\title{
Levetiracetam for Prophylactic Treatment of Pediatric Migraine: A Randomized Double- Blind Placebo-Controlled trial
}

Hadi Montazerlotfelahi ${ }^{1,}$, , Man Amanat ${ }^{2,}$, ,Ali Reza Tavasoli ${ }^{3}$, Elmira Agah ${ }^{2,4}$, Gholam Reza Zamani $^{3}$, Josemir W. Sander ${ }^{5-7}$, Reza Shervin Badv ${ }^{3}$, Mahmoud Mohammadi ${ }^{3}$, Mahdieh Dehghani ${ }^{8}$, Morteza Heidari ${ }^{3}$, Seyed Ahmad Hosseini ${ }^{9}$,Mahmoud Reza Ashrafi ${ }^{3, * *}$

1) Pediatrics Center of Excellence, Department of Pediatric Neurology, Alborz University of Medical Sciences, Karaj, Iran

2) Faculty of Medicine, Students' Scientific Research Center, Tehran University of Medical Sciences, Tehran, Iran

3) Pediatrics Center of Excellence, Department of Pediatric Neurology, Children's Medical Center, Growth and Development Research Center, Tehran University of Medical Sciences, Tehran, Iran

4) Neurolmmunology Research Association (NIRA), Universal Scientific Education and Research Network (USERN), Tehran, Iran.

5) Stichting Epilepsie Instellingen Nederland (SEIN), Achterweg 5, Heemstede 2103SW, Netherlands

6) NIHR University College London Hospitals Biomedical Research Centre, UCL Queen Square Institute of Neurology, London WC1N 3BG, and Chalfont Centre for Epilepsy, Chalfont St Peter, Bucks, SL9 ORJ, United Kingdom

7) Faculty of Medicine, Yazd University of Medical Sciences, Yazd, Iran

8) Department of Pediatric Neurology, Golestan University of Medical Sciences, Golestan, Iran

*Hadi Montazerlotfelahi and Man Amanat contributed equally to this work.

${ }^{* *}$ Corresponding Author: Mahmoud Reza Ashrafi. Pediatrics Center of Excellence, Department of Pediatric Neurology, Children's Medical Center, Growth and Development Research Center, Tehran University of Medical Sciences, Tehran, Iran E-mail: ashrafim@tums.ac.ir , Tel/fax: +98 2166935848 


\begin{abstract}
Introduction: Few drugs are available for migraine prophylaxis in children. Levetiracetam is a broad spectrum anti-seizure drug which has been suggested to be effective in reducing adult migraine episodes. We assessed the safety and efficacy of levetiracetam in the prevention of pediatric migraine. Methods: A randomized doubleblind placebo-controlled trial was performed. Eligible participants were aged 4-17 years old with at least four migraineous episodes monthly or had severe disabling or intolerable episodes. Primary endpoints were the mean changes in monthly frequency and intensity of headaches from the baseline phase to the last month of the doubleblind phase. Safety endpoint was the adverse effects reported . Results: Sixty one participants (31 taking levetiracetam and 30 taking placebo) completed the study. All had a significant reduction in frequency and intensity of episodes which was significantly greater in the levetiracetam arm. Sixty eight percent of individuals in the treatment group reported more than $50 \%$ reduction of episodes at the end of the trial compared with $30 \%$ in placebo group ( $P$-value: 0.0074 ). Irritability, day-time sedation, and mild tic were reported. Conclusion: Levetiracetam may be useful in migraine prevention and may decrease migraine episodes and severity.
\end{abstract}

Trial Registration: The study is prospectively registered with Iranian Registry of Clinical Trials; IRCT.ir, number IRCT2017021632603N1.

Key words: Anti-epileptic drug; Headache; Children; Adolescent

\title{
Introduction
}

Up to $8 \%$ of children and adolescents may have migraine (1). About half of children and adolescents with migraine will continue to experience episodes into adulthood $(2,3)$, leading to disability with substantial social and financial burdens to the individual as well as society. 
Migraine prophylaxis may reduce the burden of the condition (4). To prevent analgesic overuse and to help children have normal daily activity, migraine preventive medications should be used when the number of headache episodes exceeds four per month or when quality of life, school attendance or daily activities are restricted $(5,6)$. Few high quality studies to identify medications with reasonable prophylactic effect have been conducted in pediatric migraine. Topiramate is the only prophylactic agent of migraine in children older than 12 years approved by the Food and Drug Administration (FDA). A recent phase 3, multicenter, double-blind, placebo-controlled trial, however, suggested that topiramate was not different from placebo in reducing events in children and adolescents (7). Further work is needed to identify the best evidence-based pharmaceutical interventions.

Anti-seizure medications (ASMs) are commonly used in migraine prevention. Randomized controlled trials evaluated the efficacy of 15 ASMs as migraine prophylaxis in adults (8). Topiramate, valproic acid, levetiracetam, zonisamide and gabapentin have also been used in a few studies to prevent childhood and adolescent migraine (9). Our aim was to assess the safety and efficacy of levetiracetam in prevention of pediatric migraine.

\section{Methods}

Study design and participants 
A randomized, double-blind placebo-controlled trial in the Children's Medical Center, a major referral hospital affiliated to Tehran University of Medical Sciences was performed. The trial was divided into three phases: 1 ) initial screening phase (2 weeks), a baseline phase (4 weeks), and a double-blind treatment phase (12 weeks). Other preventive pharmacologic treatment was not allowed during the trial or in the 2 months prior to baseline (Appendix, p. 1). Use of abortive migraine medications including simple analgesics (e.g., acetaminophen, ibuprofen, ketorolac), triptan-based (e.g., sumatriptan, zolmitriptan), and ergot-derivative-based (e.g., ergotamine, dihydroergotamine) migraine medications were permitted but participants should not change the rescue drugs during the study period.

Children aged 4-17 years who met the diagnostic criteria for pediatric migraine (with or without aura) as defined by the International Headache Society (10) were enrolled. They had to have at least four migraineous episodes per month or to have severe disabling or intolerable headache. They were excluded if they had any of the following:

- History of cluster headache, hemiplegic migraine, or chronic daily headaches

- Headaches related to structural brain lesions

- Focal neurologic deficit

- No therapeutic response to at least three adequate trials of medication for headache prophylaxis

- History of levetiracetam sensitivity

- Pregnancy 
- Other neurological conditions (e.g. epilepsy)

The ethics committee of Tehran University of Medical Sciences approved the final protocol (Number: IR.TUMS.VCR.REC.1395-811). The method was explained to all involved. Parents provided written informed consent before initiation of any study procedures. The study is prospectively registered with Iranian Registry of Clinical Trials; IRCT.ir, number IRCT2017021632603N1.

Randomization and blinding

Eligible participants were randomly assigned to receive either levetiracetam or placebo in a 1:1 ratio by permuted block randomization (block sizes of four) via an interactive web response system. The responsible statistician was not involved in study conduct or monitoring. Some hospital personnel involved in the preparation of the trial material were not blinded but had no contact with participants, their parents, or investigators. The study medications were coded and administered by a nurse who was not informed about the clinical characteristics of cases. The study personnel, investigators, participants, and their parents were blinded during the course of the study until the code was broken at trial end. Packaging, size $(18 \mathrm{~mm} \times 8 \mathrm{~mm})$, shape (oval), and color (yellow) of placebo tablets were similar to the $500 \mathrm{mg}$ levetiracetam tablets used.

Procedures 
All children had complete physical examinations. Neuro-imaging was performed if clinically indicated. Laboratory screening tests including blood count (hemoglobin, white blood cells, and platelets), serum chemistry. Urine pregnancy testing were performed if appropriate (phase 1). Headache diaries were provided for each child, and the parent who had more contact with the child was responsible for recording events in the diary. A simple form of migraine diagnostic criteria (including criteria B-D) (10) translated into the Farsi language was also provided and explained to the responsible parent so that only migraineous episodes were reported. To avoid information bias, parents were asked to collect the data in real time, every day and not retrospectively. The number of migraine episodes and the intensity of the attacks were recorded using a questionnaire for a period of 4 weeks (phase 2) before initiation of double-blind phase.

\section{Phase 3}

In the 12-week double-blind phase, oral levetiracetam was administered to the treatment group. The initial dose was usually about $20 \mathrm{mg} / \mathrm{kg} /$ day divided into two doses but varied at the discretion of the study team (up to $40 \mathrm{mg} / \mathrm{kg} /$ day). Parents recorded migraine frequency and intensity daily. The first follow-up visit was one week after the initiation of treatment to check whether the medication had been taken at the correct times and doses and whether any adverse effects had occurred. Subsequent follow-up visits were at 4, 8, and 12 weeks. Participation was discontinued if 1) any adverse event 
occurred that, in the opinion of the investigator, required termination of medication; 2) there was progression of migraine attacks; 3) the participant became pregnant; or 4) consent was withdrawn.

\section{Outcomes}

The primary endpoints were the mean changes in monthly attack frequency and severity of migraine headaches from the baseline phase to the last four weeks of treatment. Migraine frequency was defined as the number of attacks that fulfilled the IHS criteria for migraine with or without aura (10). In each arm, we obtained the mean number of these episodes every four weeks after initiation of treatment.

Migraine intensity was reported using a visual analogue scale (VAS). This scale consists of a $10 \mathrm{~cm}$ line divided into 10 parts, numbered 0 (no pain) to 10 (most extreme pain). In each group, we obtained the mean of migraine intensity every four weeks after initiation of treatment.

The secondary endpoint was the $>50 \%$ responder rate. The drug was defined as effective if it decreased headache frequency by more than $50 \%$ in the double-blind phase compared with the baseline frequency.

For the safety endpoint all participants and their parents were asked to report any side effects of the medications during their monthly visits. A phone number was also 
provided so adverse events could be reported. Participants and their parents were asked to go to the emergency department if serious complications occurred.

\section{Statistical analysis}

Based on previous placebo-controlled trials in migraine, we assumed a $40 \%$ reduction in migraine attack frequency with standard deviation (SD) $50 \%$ for sample size calculation. Power analysis mandated at least 25 cases per group (11) with $\alpha$ (the probability of type I error) as 0.05 , and $\beta$ (the probability of type II error $(1-$ power of the test)) as 0.20 . Considering a $20 \%$ dropout rate, a final sample size of 30 subjects in each treatment arm was estimated. The formula used for sample size calculation was:

$$
\frac{\left(\sigma_{1}^{2}+\sigma_{2}^{2}\right)\left(z_{1-\frac{\alpha}{2}}+z_{1-\beta}\right)^{2}}{\Delta^{2}}
$$

$\sigma:$ Variance of mean; $\Delta$ : Absolute difference between two means; $z$ : Critical value for a given $\alpha$ or $\beta$

Statistical analysis was performed by the statistician who was masked to the study arms. Continuous variables were reported as mean with SD, standard error mean (SEM), or $95 \%$ confidence interval $(\mathrm{Cl})$. Categorical variables were reported using percentages. Analyses of between-group differences were performed using intention to treat (ITT) approach. All individuals randomized who received at least one dose of the study drug were included. Missing data was handled by multiple imputation. Secondary analyses were also performed for participants who completed the trial. 
The mean change in frequency and intensity of migraine attacks during weeks 9-12 were compared between the two treatment arms using t-test for two independent samples. To more rigorously evaluate the robustness of the treatment effect, analysis of covariance (ANCOVA) models with adjustment for covariates including age, gender, and baseline migraine frequency or intensity was performed as a supplemental analysis. Fisher's exact test was used for the secondary endpoint ( $>50 \%$ responder rate) analysis. Participants who failed to complete the study were considered nonresponders. IBM SPSS Statistics version 25 was used for statistical analyses. Figures were made using GraphPad Prism version 7.04.

\section{Results}

\section{Participants}

The initial phase started in July 2017. Double-blind treatment was given from August 2017 when the first participant was assigned to August 2018. Eighty-six individuals were screened for eligibility, of whom 68 were eligible and were randomly assigned to a treatment group (34 in each group). Each took at least one dose of the allocated drug and was therefore eligible for the ITT analysis. Of these 68 included subjects, three receiving levetiracetam and four receiving placebo did not complete the study. Reasons for drop-out were: lost to follow-up ( $n=3)$, poor compliance $(n=3)$, and withdrew consent without providing any specific reason $(n=1)$. No adverse event was reported by these participants. Overall 61 individuals (31 on levetiracetam) completed the study (Figure 1). The baseline characteristics of the participants are summarized in Table 1 and Figure 2 . 
Seven subjects were included due to severely disabling migraineous attacks (2 taking levetiracetam and 5 taking placebo) and the rest had at least four migraine episodes per

month. At least one other prophylactic medication had been unsuccessful in 8 participants taking levetiracetam and 9 taking placebo while 51 individuals had no history of previous preventive treatment. Simple analgesics were the most commonly used acute migraine treatment in both arms (Supplementary Table 1). For individuals who received levetiracetam, doses ranging from $500 \mathrm{mg}$ to $3000 \mathrm{mg}$ per day were administered (Mean $\pm S D$ : $1120.9 \pm 487 \mathrm{mg})$.

\section{Primary endpoints}

During the last four weeks of the double-blind phase, the mean migraine frequency reduced significantly in the levetiracetam and placebo arms compared to baseline (Figure 3). The mean reduction of migraine frequency was statistically greater in levetiracetam than in the placebo arm $(95 \% \mathrm{Cl}:-6.54$ to -2.13$)$ (Table 2). The mean migraine intensity also reduced significantly in both treatment groups during the last four weeks of study period compared to baseline (Figure 3), but the difference was significantly greater in those taking levetiracetam $(95 \% \mathrm{Cl}:-3.04$ to -0.38$)$ (Table 2$)$.

Using the ANCOVA sensitivity analysis, the treatment effect defined by the primary endpoints remained significant and migraine frequency and intensity of participants taking levetiracetam had greater reduction than cases taking placebo (migraine frequency [mean difference $(95 \% \mathrm{Cl})$ ]: $-2.84(-4.52$ to -1.17$)$; migraine intensity [mean difference $(95 \% \mathrm{Cl})]$ : $-1.68(-2.95$ to -0.41$))$. 
Exploratory analyses of between-group differences in mean migraine frequency and intensity during the first and second four week periods of the double-blind phase also showed greater reductions in the levetiracetam arm than in the placebo arm (Supplementary Table 2).

\section{Secondary endpoint}

Levetiracetam was associated with a greater than $50 \%$ reduction from baseline migraine episodes in 17 (50\%) participants at four weeks after initiation of medication; $19(56 \%)$ after eight weeks, and 21 (62\%) after 12 weeks. Six of the participants in the levetiracetam arm reported complete elimination of headaches. Conversely, $6(18 \%)$ of those in the placebo arm had a greater than $50 \%$ reduction from their baseline frequency at four weeks after the beginning of double-blind treatment; $8(24 \%)$ after eight weeks, and 9 (26\%) after 12 weeks. One had a complete cessation of headache attacks. Overall, the $>50 \%$ responder rate was significantly greater in the levetiracetam arm than the placebo $(\mathrm{p}=0.007)$ (Table 2$)$.

\section{Safety endpoint}

Adverse events occurred in eight individuals taking levetiracetam. None was significant and did not lead to discontinuation of therapy. Overall, irritability (four cases), day-time sedation (two cases), and mild tic (two cases) were reported, which all resolved during treatment. No side effect was reported in participants receiving placebo.

\section{Safety endpoint}


Adverse events occurred in eight individuals taking levetiracetam. None was significant and did not lead to discontinuation of therapy. Overall, irritability (four cases), day-time sedation (two cases), and mild tic (two cases) were reported, which all resolved during treatment. No side effect was reported in participants receiving placebo.

\section{Discussion}

\section{Summary of results}

Levetiracetam and placebo significantly reduced migraine frequency and intensity compared to baseline. The reduction in the levetiracetam arm was, however, significantly greater than with placebo. Over two thirds of participants in the treatment arm reported a reduction of more than 50\% from baseline headaches with levetiracetam which was significantly greater than with placebo. More participants reported adverse events on levetiracetam but safety was similar to placebo.

The annual health-care costs of children diagnosed with migraine are three-fold greater than controls (12). These individuals are also prone to developing different neurological and mental co-morbidities in the future (13). About $18 \%$ of participants (with baseline mean migraine frequency of 12 per month) became headache-free after 12 weeks of treatment with levetiracetam. Migraine episodes only stopped in one individual on placebo. This difference can be significant from a clinical standpoint. 
Previous studies

A full search identified six studies (14-19) (Table 3); all suggested that levetiracetam could significantly reduce headache frequency. An open-label prospective study (14) showed that $90 \%$ of people with migraine had a greater than $50 \%$ reduction in headache frequency while around two-third of our participants in the levetiracetam group showed this significant decrease. This discrepancy could possibly be due to methodological differences. None of the above-mentioned studies used a control group to compare the efficacy of levetiracetam to placebo. In line with this trial, irritability was the commonest side-effect in other studies. Overall, irritability, somnolence, dizziness, memory defects, weight gain, sleep disturbance and delusion were recorded in studies (14-18). These side-effects led to discontinuation of therapy in three cases from two studies $(14,18)$.

Levetiracetam was also used as a prophylactic agent for episodic and chronic migraine in adults. Prospective double-blind placebo-controlled trials showed that this medication produced significant reductions in migraine episodes, as well as attack severity, in adult episodic migraine $(20,21)$. One trial showed that individuals in levetiracetam and valproate groups had more improvements in headache frequency than those in the placebo arm but no significant difference was reported when compared to each other (21). Randomized double-blind studies conducted in adults with chronic migraine did not show significant efficacy of levetiracetam compared to placebo (22) or valproate (23). 
Side effects including irritability, somnolence, dizziness, hyperactive behavior, moodiness, and hostility were also reported in adults (21).

\section{Levetiracetam mechanisms of action}

The exact mechanism by which levetiracetam acts is unknown but it probably has unique mechanisms of action, binding to the synaptic vesicle protein (SV2A) leading to changes in the release of numerous neurotransmitters (24).

To date, levetiracetam and many otherASMs (e.g. topiramate) have been used in human and animal studies for prevention of migraine but the underlying mechanisms of efficacy are yet unclear. The pathophysiologic link between migraine and epilepsy may resolve some of our ambiguities. Increased prevalence of migraine among individuals with epilepsy as well as of epilepsy in people with migraine (25) suggests possible shared pathophysiology between these conditions. Similarities in clinical features of epilepsy and migraine such as presence of premonition, aura, and post-ictal phases may suggest elements of pathophysiologic overlay (26) Immune system dysfunctions including elevated levels of pro-inflammatory cytokines and autoantibodies are now considered in occurrence of epilepsy and migraine $(27,28)$.

Placebo effects 
Our results suggest that placebo could also be useful in improving pediatric migraine. A recent trial suggested that placebo was more efficacious than topiramate or amitriptyline in producing at least $50 \%$ reduction in headache-days in children and adolescents (7). High placebo-response rates have also been reported in previous headache and pain trials (reviewed in 29,30). Placebo effects are neurobiologic changes that may occur due to expectations of people with migraine, their caregivers and clinicians (31).

\section{Limitations and future directions}

The small sample size, short follow-up period, and lack of active comparator are major limitations of this trial which should be resolved in future studies. As migraine cannot be objectively assessed the outcomes of the study were reliant on reports from participants or their parents which could lead to information bias. Multiple factors including diet, physical activity, and stressors affect migraine occurrence and further studies should estimate their impacts on results. Future trials are needed to suggest the most effective preventive medications in pediatric migraine. Trying to understand the pathophysiologic links between migraine and its co-morbidities may also help find new treatments for migraine. Future studies should also identify subgroups that may have the most benefits from levetiracetam and other ASMs.

Conclusion 
Levetiracetam was more successful than placebo in decreasing migraine episodes and their intensity. It was also more effective than placebo in reducing headache frequency by $50 \%$ in participants in a 12 weeks period.

\section{Funding}

The trial was funded by The Research Deputy of Tehran University of Medical Sciences (grant number: 95-01-30-31762), who had no role in study design, data collection, analysis, or interpretation of data, in the writing of the report, or in the decision to submit the article for publication.

\section{Ethics approval}

The ethics committee of the Tehran University of Medical Sciences approved this study (Number: IR.TUMS.VCR.REC.1395-811).

\section{Study protocol}

The study is registered with Iranian Registry of Clinical Trials; IRCT.ir, number IRCT2017021632603N1. The study protocol can be observed in Appendix, p. 5,6.

\section{Acknowledgements}

We are grateful to Cobel Darou for providing levetiracetam and placebo. We are also very grateful to all parents and participants for partaking in this study. Our special thanks to Mrs Leila Forotan who dispensed the trial medications to the participants. JWS is based at the NIHR University College London Hospitals Biomedical Research 
Centre, which receives a proportion of funding from the UK Department of Health's Research Centers funding scheme. He receives research support from the Dr Marvin Weil Epilepsy Research Fund and from the UK Epilepsy Society.

\section{Conflicts of interest}

JWS has received research funding from Eisai and UCB, personal fees from Eisai, UCB, GW and Zogenix Pharma, outside the submitted work. The other authors declare no competing interests. 


\section{Clinical Implications:}

- Few drugs are available in migraine prophylaxis of pediatric population

- Levetiracetam may be effective in pediatric migraine prevention

- Levetiracetam may reduce the number and intensity of migraine episodes; this needs replication in larger groups. 


\section{References}

1) Abu-Arafeh I, Razak S, Sivaraman B, Graham C. Prevalence of headache and migraine in children and adolescents: A systematic review of population-based studies. Dev Med Child Neurol 2010; 52:1088-1097.

2) Bille B. A 40-year follow-up of school children with migraine. Cephalalgia 1997; 17:488-491.

3) Virtanen R, Aromaa M, Rautava P, Metsähonkala L, Anttila P, Helenius H, Sillanpää M. Changing headache from preschool age to puberty. A controlled study. Cephalalgia 2007; 27:294-303.

4) D'amico D, Solari A, Usai S, Santoro P, Bernardoni P, Frediani F, De Marco R, Massetto N, Bussone G, for the Progetto Cefalee Lombardia Group. Improvement in quality of life and activity limitations in migraine patients after prophylaxis. A prospective longitudinal multicentre study. Cephalalgia 2006; 26:691-696.

5) Lewis DW. Pediatric migraine. Neurol Clin 2009; 27:481-501.

6) Damen L, Bruijn J, Verhagen AP, Berger MY, Passchier J, Koes BW. Prophylactic treatment of migraine in children. Part 2. A systematic review of pharmacological trials. Cephalalgia 2006; 26:497-505.

7) Powers SW, Coffey CS, Chamberlin LA, Ecklund DJ, Klingner EA, Yankey JW, Korbee LL, Porter LL, Hershey AD. Trial of amitriptyline, topiramate, and placebo for pediatric migraine. N Eng J Med 2017; 376:115-124.

8) Mulleners WM, McCrory DC, Linde M. Antiepileptics in migraine prophylaxis: an updated Cochrane review. Cephalalgia 2015; 35:51-62. 
9) Kacperski J. Prophylaxis of migraine in children and adolescents. Pediatr Drugs $2015 ; 17: 217-226$.

10) Olesen J. Headache Classification Sub-Committee of the International Headache Society. The International Classification of Headache Disorder, 2nd edn. Cephalagia $2004 ; 24: 1-160$

11) Kane SP. Sample Size Calculator. ClinCalc:

http://clincalc.com/stats/samplesize.aspx. (Updated July 1, 2017. Accessed May 22, 2017)

12) Pesa J, Lage MJ. The medical costs of migraine and comorbid anxiety and depression. Headache $2004 ; 44: 562-570$.

13) Hamelsky SW, Lipton RB. Psychiatric comorbidity of migraine. Headache 2006; 46:1327-1333.

14) Miller GS. Efficacy and safety of levetiracetam in pediatric migraine. Headache $2004 ; 44: 238-243$.

15) Pakalnis A. Levetiracetam prophylaxis in pediatric migraine-an open-label study. Headache 2007; 47:427-430.

16) Awaad Y, Rizk T. Levetiracetam in the treatment of pediatric headache. JTU Med Sc 2014; 9:74-77.

17) Sediqi M, Yavari B, Almasi A. Prophylactic efficacy of levetiracetam on migraine headaches in children aged 4-14-year-old. Ann Trop Med Public Health 2017; 10:1547. 18) Vaisleib II, Neft RA, Schor NF. Role of levetiracetam in prohylaxis of migraine headaches in childhood. Neurology 2005; 64:A-343.

19) DeSouza TG, Shahid A, Waren SP. The use of levetiracetam in the prophylactic treatment of migraine headaches in adolescents. Headache $2005 ; 45: 819-820$ 
20) Verma AA. Levetiracetam in migraine prophylaxis: a randomized placebocontrolled study in a rural medical institute in northern India. Clin Neuropharmacol 2013; 36:193-197.

21) Sadeghian H, Motiei-Langroudi R. Comparison of levetiracetam and sodium valproate in migraine prophylaxis: a randomized placebo- controlled study. Ann Indian Acad Neurol 2015; 18:45-48.

22) Beran RG, Spira PJ. Levetiracetam in chronic daily headache: a double- blind, randomised placebo-controlled study. (The Australian KEPPRA Headache Trial [AUSKHT]). Cephalalgia 2011; 31:530-536.

23) Kashipazha D, Ghadikolaei HS, Siavashi M. Levetiracetam in compare to sodium valproate for prophylaxis in chronic migraine headache: a randomized double-blind clinical trial. Curr Clin Pharmacol 2017; 12:55-59.

24) Lynch BA, Lambeng N, Nocka K, Kensel-Hammes P, Bajjalieh SM, Matagne A, Fuks B. The synaptic vesicle protein SV2A is the binding site for the antiepileptic drug levetiracetam. Proc Natl Acad Sci U S A 2004; 101:9861-9866.

25) Keezer MR, Sisodiya SM, Sander JW. Comorbidities of epilepsy: current concepts and future perspectives. Lancet Neurol 2016; 15:106-115.

26) Nye BL, Thadani VM. Migraine and epilepsy: review of the literature. Headache $2015 ; 55: 359-380$.

27) Amanat M, Thijs R, Salehi M, Sander JW. Seizures as a clinical manifestation in somatic autoimmune disorders. Seizure 2019; 64:59-64.

28) Yılmaz IA, Özge A, Erdal ME, Edgünlü TG, Çakmak SE, Yalın OÖ. Cytokine polymorphism in patients with migraine: some suggestive clues of migraine and inflammation. Pain Med 2010; 11:492-497. 
29) Evers S, Marziniak M, Frese A, Gralow I. Placebo efficacy in childhood and adolescence migraine: an analysis of double-blind and placebo-controlled studies. Cephalalgia 2009; 29:436-444.

30) El-Chammas K, Keyes J, Thompson N, Vijayakumar J, Becher D, Jackson JL. Pharmacologic treatment of pediatric headaches: a meta-analysis. JAMA Pediatr 2013; $167: 250-258$

31) Powers SW, Coffey CS, Chamberlin LA, Ecklund DJ, Klingner EA, Yankey JW, Korbee LL, Porter LL, Hershey AD. Trial of amitriptyline, topiramate, and placebo for pediatric migraine. N Eng J Med 2017; 376:115-124. 
Figure 1. CONSORT diagram of trial flow

Figure 2. Age distribution of study participants

Figure 3. Mean of migraine frequency per 4 weeks

Figure 4. Mean of migraine intensity per 4 weeks 